www.nature.com $/ \mathrm{hr}$

\title{
Performance of body fat and body mass index cutoffs in elevated blood pressure screening among male children and adolescents
}

\author{
Romulo A Fernandes ${ }^{1,2}$, Diego GD Christofaro ${ }^{2,3}$, Camila Buonani ${ }^{4}$, Henrique L Monteriro ${ }^{1}$, \\ Jefferson R Cardoso ${ }^{5}$, Ismael F Freitas $\mathrm{Jr}^{4}$ and Dalmo RL Machado ${ }^{6}$
}

For percentage of body fat (\%BF), there are no internationally accepted cutoffs. The primary function of body fat cutoffs should be to identify not only excessive body fatness, but also the increased risk of unhealthy outcomes, such as hypertension. The purpose of this study was to analyze the accuracy of different \%BF and body mass index (BMI) cutoffs as screening measures for EBP in pediatric populations. It was a cross-sectional study with a sample of 358 male subjects from 8 to 18 years old. BP was measured by the oscilometric method, and body composition was measured by dual-energy X-ray absorptiometry (DXA). The accuracy of three reference tables used for body fat cutoffs was assessed. The three body fat reference tables were highly specific, but insensitive, for elevated BP screening. For elevated BP screening, all body fat cutoffs presented similar sensitivity (range $=48.3-53.7 \%$ ) and specificity (range $=79.2-84.1 \%$ ). The body fat cutoffs performed no better than BMI in screening of children and adolescents at risk of elevated BP (EBP). BMI seems a more attractive tool for this function, as it performed similarly and can be applied in large surveys and with lower costs.

Hypertension Research (2011) 34, 963-967; doi:10.1038/hr.2011.61; published online 26 May 2011

Keywords: adolescent; children; dual-energy X-ray absorptiometry; obesity; receiver operating characteristic curve

\section{INTRODUCTION}

The increase of worldwide obesity among pediatric populations in recent decades has been identified in both developed and developing countries. ${ }^{1}$ This increase is worrisome, because it is expressly associated with a higher burden of health care costs among children and adolescents. $^{2}$

Adipose tissue releases a variety of adipokines, which are strongly associated with endothelial dysfunction and, in turn, increased blood pressure (BP) values at rest. It is known that obese adolescents have increased odds of developing chronic disorders such as hypertension, ${ }^{3}$ and this physiological outcome is frequently observed among obese adolescents. $^{3-7}$ Therefore, the primary function of body fat cutoffs should be to screen not only for excessive body fatness, but should also be to asses the increased risk of unhealthy outcomes, such as hypertension.

Williams et al. ${ }^{8}$ proposed percentage of body fat (\%BF) cutoffs for white and black American children and adolescents (5-18 years old), based on values of cardiovascular risk factors (with \%BF based on skinfold measurements). Taylor et al. ${ }^{9}$ went further by utilizing a reference method for the assessment of body composition (dual- energy X-ray absorptiometry (DXA)) and proposed sex- and agespecific \% BF cutoffs among white New Zealanders (3-18 years old), but these were not associated with indicators of cardiovascular risk. More recently, McCarthy et al., ${ }^{10}$ in a large Caucasian British pediatric sample, proposed the 85th and 95th percentile of \%BF (sex- and age-specific cutoffs (5-19 years old)) to define 'overfat' and obesity (with \%BF, based on bioelectric impedance analysis), respectively.

It is possible that this large variety of cutoffs proposed for children and adolescents could exert considerable negative influence on the screening of unhealthy outcomes associated with obesity. Nevertheless, information about this question is not available in the literature. Furthermore, it is not clear if anthropometric indices (for example, body mass index (BMI) and waist circumference), which are cheaper and more routinely used in clinical and epidemiological settings, screen subjects with increased risk of elevated blood pressure (EBP) more or less adequately than \%BF.

Thus, the purposes of this study were: (i) to analyze the influence of cutoff choice on the accuracy of body fat estimation as a screening tool for EBP in male pediatric populations and (ii) to verify if BMI presents similar screening characteristics to these body fat cutoffs.

${ }^{1}$ Department of Physical Education, UNESP Univ Estadual Paulista, Institute of Bioscience, Rio Claro, Brazil; ${ }^{2}$ Department of Physical Education, Universidade do Oeste Paulista UNOESTE, Presidente Prudente, Brazil; ${ }^{3}$ Department of Collective Health, Universidade Estadual de Londrina UEL, Londrina, Brazil; ${ }^{4}$ Department of Physical Education, UNESP Univ Estadual Paulista, Presidente Prudente, Brazil; ${ }^{5}$ Department of Physical Therapy, Universidade Estadual de Londrina UEL, Londrina, Brazil and ${ }^{6}$ Department of Physical Education, Universidade de Sao Paulo USP, Ribeirao Preto, Brazil

Correspondence: Professor RA Fernandes, Department of Physical Education. Institute of Biosciences-UNESP, Av 24, 1515. 13506-900 Rio Claro (SP), Brazil.

E-mail: romulo_ef@yahoo.com.br

Received 27 March 2010; revised 7 December 2010; accepted 23 March 2011; published online 26 May 2011 


\section{METHODS}

\section{Sample}

This cross-sectional study was carried out in the city of Presidente Prudente (Human Development Index $=0.846$ ), Southeastern Brazil, from July to November 2008. Before the study, a minimum required sample size was estimated $(n=275)$, based on an obesity prevalence of $13.9 \%,{ }^{1}$ an error of $5 \%$, an $\alpha$-error of $5 \%(z=1.96)$ and a design effect of 1.5 . Furthermore, an additional loss rate of $10 \%$ was considered $(n=303)$. After the fieldwork, the sample included 358 male children and adolescents from 8 to 18 years old ( $n=92$ children (from 8 to 10 years old) and $n=266$ adolescents (from 11 to 18 years old)). The sample population was found at schools $(n=3)$ and recreational associations $(n=3)$, where all male students/members in each setting were invited to participate as volunteers in the study. Inclusion criteria for participants consisted of a self-declaration of health and an affirmation that they were neither taking any prescription medicine nor undergoing any current clinical treatment. In each school or club, all interested students received a written informed consent form, which was to be filled out by both the parents and the students before they could take part in the study. The study was approved by the Ethics Committee on Human Experimentation of the Universidade de São Paulo.

\section{Anthropometric measures}

Body weight (wearing shorts and a t-shirt (nearest $100 \mathrm{~g}$ )) and barefoot height were measured with an electronic scale and wall-mounted stadiometer, respectively. BMI was calculated with values of weight divided by height, squared $\left(\mathrm{kg} \mathrm{m}^{-2}\right)$. All anthropometry was assessed using standardized techniques ${ }^{11}$ by a single experienced researcher. Children and adolescents were classified as overweight or obese according to the BMI-based definition proposed by Cole et al. ${ }^{12}$ (in all statistical analysis, overweight and obesity were merged).

\section{Blood pressure (BP) values}

Systolic and diastolic blood pressure (SBP and DBP) values were measured with an electronic device (MX3 Plus; Omron, Kyoto, Kansai, Japan) previously validated for pediatric populations. ${ }^{13}$ After 5 min of rest in a seated position, two measures were taken on the right arm, with a 2 min interval between them, and the mean value was used. For the subjects' blood pressure (BP) measurement, two types of cuffs were used according to the arm circumference $(6 \mathrm{~mm} \times 12 \mathrm{~mm}$ for children, and $9 \mathrm{~mm} \times 18 \mathrm{~mm}$ for adolescents $14-18$ years old and for those children with a larger arm size), following the recommendations of the American Heart Association. ${ }^{14}$ The National High Blood Pressure Education Program ${ }^{15}$ cutoffs adjusted for age and height percentile (P95), were applied to indicate the presence of elevated BP (EBP).

\section{Dual-energy X-ray absorptiometry (DXA)}

Whole body and regional body composition were measured with a Lunar DPXNT scanner (Lunar DPX-NT; General Electric Healthcare, Little Chalfont, Buckinghamshire, UK). The software provides measurements of lean soft tissue, fat and bone mineral density for the entire body, as well as for specific regions, as well as \%BF. All DXA measurements were made at a university laboratory with a constant controlled temperature. Each morning, before any measurements were taken, the device was calibrated by the same researcher and, according to the reference values provided by the manufacturer, the tests presented high reliability.

\section{Body fat cutoffs}

The comparison of body fat cutoffs was based on three reference tables developed for children and adolescents of different nations (no cutoff was adjusted according to ethnic groups): (i) Williams et al..$^{8}$ classify subjects as either non-obese or obese (American population); (ii) Taylor et al. ${ }^{9}$ use nonobese, overweight and obese as categories, in which the categories overweight and obese were clustered (overweight+obesity; New Zealand population); whereas (iii) McCarthy et al. ${ }^{10}$ use the following classifications: 'normal', 'overfat' and 'obese', in which the categories overfat and obese were clustered (overfat+obesity; British population).

\section{Statistical analysis}

Initially, the Kolmogorov-Smirnov test rejected the normality of the numerical data, and so, non-parametric descriptive statistical methods were applied (median and interquartile range). Additionally, the Mann-Whitney $U$-test was used to compare numerical variables between two groups (subjects with normal and elevated BP values). Spearman's rank was used to analyze correlations between BP values and chronological age. The kappa $(\kappa)$ statistic indicated the agreement level between categorical variables. The chi-square $\left(\chi^{2}\right)$ test, either with Yates' correction or linear trend analysis, was used to determine associations among categorical outcomes. Receiver operating characteristics curves with the default parameters (sensitivity (true-positive rate), specificity (true-negative rate), positive predictive value, negative predictive value and area under curve (AUC)) were compared, to determine the best $\% \mathrm{BF}$ cutoff reference table. Significance $(P)$ was set at $5 \%$ and statistical software SPSS version 13.0 (SPSS, Chicago, Il, USA) was utilized for all analyses.

\section{RESULTS}

The sample was composed of 358 male children and adolescents. The prevalence of EBP was $26 \%(n=93)$; chronological age was poorly correlated to BP values (SBP: $r=0.38$ with $P=0.001$; DBP: $r=0.22$ with $P=0.001$ ). Table 1 shows the general characteristics of the sample grouped by BP values. Subjects with EBP had higher values of body weight, $\mathrm{BMI}$ and $\% \mathrm{BF}$. Independent of \%BF cutoff, obese subjects presented higher values of both SBP and DBP (all with $P=0.001$ ). Children and adolescents who were identified as overweight/obese by BMI also presented higher values of SBP and DBP than those with normal weight (both comparisons with $P=0.001$ ).

The three cutoffs for body fat presented elevated agreement in the identification of obese children and adolescents (Taylor et al. ${ }^{9}$ and McCarthy et al. ${ }^{10}(\mathrm{k}=0.93 ; P=0.001)$; Taylor et al.$^{9}$ and Williams et al. ${ }^{8}$ $(\mathrm{k}=0.87 ; P=0.001)$; Williams et al. ${ }^{8}$ and McCarthy et al. ${ }^{10}(\mathrm{k}=0.89$; $P=0.001)$ ), whereas, BMI presented moderate agreement in the identification of obesity (Taylor et al. ${ }^{9} \quad(\mathrm{k}=0.62 ; P=0.001)$; McCarthy et al..$^{10}(\mathrm{k}=0.58 ; P=0.001)$; Williams et al. $\left.{ }^{8}(\mathrm{k}=0.60 ; P=0.001)\right)$. The prevalence of obesity indicated by Williams et al's ${ }^{8}$ cutoffs was not lower in either adolescents or children $(P=0.376)$. Taylor et al's ${ }^{9}$ cutoffs were associated with age groups (children $=40.2 \%$ and adolescents $=26.5 \% ; P=0.003$ ), whereas McCarthy et al $\mathrm{s}^{10}$ cutoffs were not associated with age $(P=0.126)$. Figure 1 shows the prevalence of obesity identified by DXA and overweight/obesity identified by BMI. There was no significant difference between the obesity prevalence identified by Williams et al's, ${ }^{8}$ Taylor et al's ${ }^{9}$ and McCarthy et al's ${ }^{10}$ cutoffs. Independently of $\% \mathrm{BF}$ cutoffs, there was a positive association between EBP values and obesity $(P=0.001$, for all), because obese subjects presented a higher occurrence of EBP. The same pattern of association was observed between BMI status and prevalence of EBP. Regarding EBP rate, there were no differences among any of the

Table 1 General characteristics of the sample grouped according BP values

\begin{tabular}{lccc}
\hline & Normal BP $(\mathrm{n}=265)$ & $E B P(\mathrm{n}=93)$ & \\
Variable & Median $(I R)$ & Median $(I R)$ & P-value \\
\hline Age (years) & $12.8(5.2)$ & $13.6(5.2)$ & 0.468 \\
Weight (kg) & $43.5(25.9)$ & $57.5(26.1)$ & 0.001 \\
Height (cm) & $154.1(31.1)$ & $161.7(27.7)$ & 0.103 \\
BMI ( $\left.\mathrm{kg} \mathrm{m}^{-2}\right)$ & $17.8(4.1)$ & $20.9(5.3)$ & 0.001 \\
\%BF & $13.4(9.1)$ & $22.1(19.2)$ & 0.001
\end{tabular}

Abbreviations: BP, blood pressure; \%BF, body fat percentage; BMI, body mass index; IR, interquartile range. 


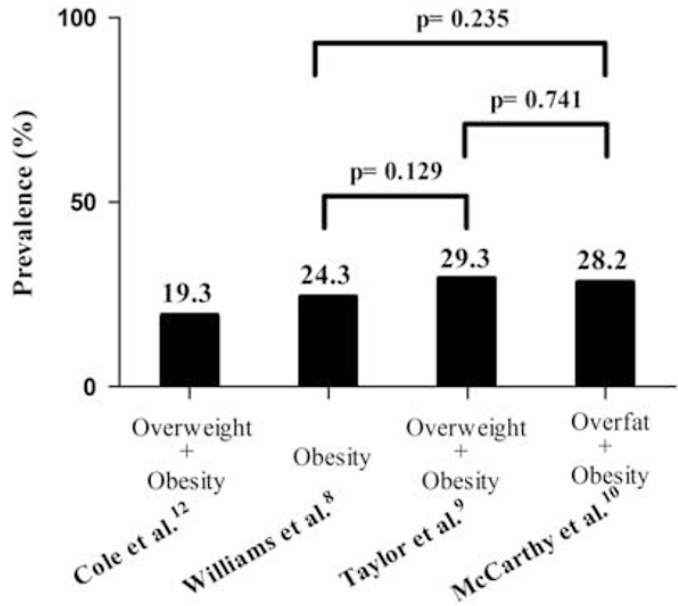

Figure 1 Obesity prevalence in male children and adolescents according to various body fat cutoffs.

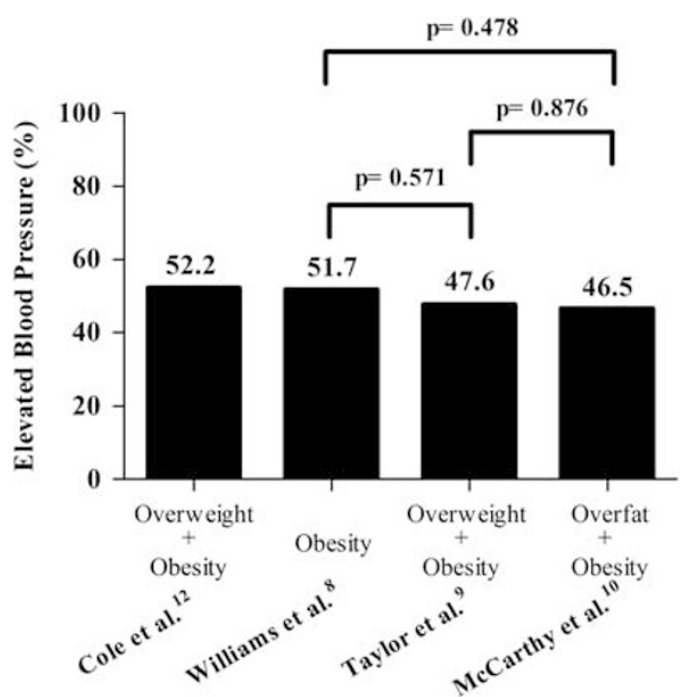

Figure 2 Elevated blood pressure (EBP) prevalence in male children and adolescents according to various body fat cutoffs and body mass index (BMI).

body fat references (Figure 2) or between body fat cutoffs and BMI cutoffs (data not shown).

BMI and \%BF are presented as screening tests for EBP (Table 2). Sensitivity and specificity were similar for all body fat and BMI cutoffs in the studied samples. Independently of cutoff, the proportion of true-positive cases was low and ranged from 52 to $70 \%$ and from 34 to $48.5 \%$ in children and adolescents, respectively. Compared with the true-positive cases, true-negative cases were much higher (ranging from 79.2 to $87.5 \%$ ). Regarding EBP screening, there were no differences among any of the body fat references or between body fat cutoffs and BMI cutoffs ( $\chi^{2}$-test with $P>5 \%$ for all comparisons).

The rate of misclassification was around of $30 \%$ in all body fat and BMI cutoffs (Williams et al. ${ }^{8}=25.1 \%(n=90)$, Taylor et al. ${ }^{9}=27.3 \%$ $(n=98)$, McCarthy et al. ${ }^{10}=27.9 \%(n=100)$ and Cole et al. ${ }^{12}=25.1 \%$ $(n=90))$. BMI presented the lowest proportion of false-positive cases $(36.6 \%(n=33))$ followed by Williams et al. ${ }^{8}(46.6 \% \quad(n=42))$, McCarthy et al. ${ }^{10}(54 \%(n=54))$ and Taylor et al. ${ }^{9}(56.1 \%(n=55))$.
However, Taylor et al. ${ }^{9}$ presented the lowest proportion of falsenegative cases $(43.9 \%(n=43))$, followed by McCarthy et al. ${ }^{10}(46 \%$ $(n=46))$, Williams et al. ${ }^{8}(53.4 \%(n=48))$ and Cole et al. ${ }^{12}(63.3 \%$ $(n=57))$. The proportion of efficiency also was similar for all reference tables ( $P>5 \%$ for all comparisons). Figure 3 shows the global measure of screening accuracy of the analyzed cutoffs.

\section{DISCUSSION}

This cross-sectional study analyzed the accuracy of three \%BF cutoffs in EBP screening among male children/adolescents, and presented data which suggests that the choice of \%BF cutoffs does not have a significant influence on such screening. When compared with these cutoffs, BMI produces similar screening characteristics.

The EBP rate found in this study ( $\sim 26 \%$ ) was higher than the $10 \%$ observed among the general Brazilian pediatric population. ${ }^{16}$ This may have been partially due to the obesity observed in a large percentage of the overall sample. Increased white adipose tissue releases a variety of adipokines that are related to a decrease in the production and utilization of nitric oxide, which has important functions in the control of vascular tone and suppression of vascular smooth muscle cell proliferation. This decreased effect of nitric oxide is associated with endothelial dysfunction and, thus, arterial hypertension. ${ }^{17}$ In pediatric populations, previous studies have found that high SBP is frequently related to higher adiposity, ${ }^{3,16}$ indicating that the above-mentioned mechanisms could occur not only in adults, but also in children and adolescents.

Obesity identified by DXA (independent of cutoff) and BMI were markedly associated with EBP. However, the results seem to indicate that the magnitude of this association is slightly cutoff dependent (Williams et al's ${ }^{8}$ cutoffs, odds ratio=4.7 (95\% confidence intervals $=2.9 ; 8.4)$; McCarthy et al ${ }^{10}$ cutoffs, odds ratio=4.4 $(95 \%$ confidence intervals $=2.6 ; 7.3$ ); Taylor et al's ${ }^{9}$ cutoffs, odds ratio $=4.0$ $(95 \%$ confidence intervals $=2.4 ; 6.6)$ ). This data indicate that $\% \mathrm{BF}$ cutoff choice has no significant effect when testing the association between obesity and EBP.

In the McCarthy et al. ${ }^{10}$ table (85th percentile), the body fat range was from 20.1 to $23 \%$, which was similar to the range observed among adolescents in New Zealand, from 18 to $23 \%$ (overweight in the Taylor et al's ${ }^{9}$ cutoffs). In contrast to the observations of Williams et al. ${ }^{8}$ (fixed at $25 \%$ for all ages), the McCarthy et al. ${ }^{10} \% \mathrm{BF}$ cutoffs showed a different pattern across age groups. Maturational and growth-related changes have great importance in this period of human development. Fernandes et al. ${ }^{18}$ proposed \%BF cutoffs based on EBP, which varied across age groups, indicating that growth represents an important confounding factor and must be taken into consideration. Thus, the absence of similarity for these cutoffs would seem to discourage the establishment of a single \%BF cutoff across different ages.

For EBP screening in pediatric populations, the utilization of internationally accepted standard reference tables for \%BF, adjusted by age groups, should receive wider acceptance, as has happened with the BMI. ${ }^{12}$ However, as is true for the BMI, the adoption of international reference tables seems to increase the proportion of falsenegative cases. ${ }^{19-21}$ This is the case with the non age-adjusted cutoffs proposed by Williams et al. ${ }^{8}$ where the higher cutoffs generated lower prevalence of obesity and, hence, higher specificity (true-negative cases). As mentioned above, outcome underestimation generates lower sensitivity (true-positive cases), that is, the capacity to correctly identify the outcome presence; therefore, both Taylor et al's ${ }^{9}$ and McCarthy et al's ${ }^{10}$ cutoffs, which presented higher outcome prevalence, also demonstrated slightly better accuracy in identifying the 
Table 2 Accuracy of BMI and body fat cutoffs in screening of EBP in male children and adolescents

\begin{tabular}{|c|c|c|c|c|c|c|c|c|c|}
\hline \multirow[b]{2}{*}{ Age groups } & \multirow[b]{2}{*}{ Cutoffs } & \multicolumn{4}{|c|}{ Parameters of the ROC curves } & \multirow[b]{2}{*}{ Efficiency $^{b}$} & \multicolumn{3}{|c|}{ Comparison with BMI (P-value $)^{\mathrm{a}}$} \\
\hline & & SENS & $S P E C$ & PPV (\%) & NPV (\%) & & SENS & SPEC & Efficiency \\
\hline \multirow{3}{*}{ Children ( $n=92)$} & Taylor et al. ${ }^{9}$ & $16(69.5 \%)$ & $48(69.5 \%)$ & 43.2 & 87.2 & $64(69.5 \%)$ & 0.413 & 0.374 & 0.749 \\
\hline & McCarthy et al. ${ }^{10}$ & $13(56.5 \%)$ & $52(75.3 \%)$ & 43.3 & 83.8 & $65(70.6 \%)$ & 0.830 & 0.765 & 0.871 \\
\hline & Cole et al. ${ }^{12}$ & $12(52.1 \%)$ & $54(78.2 \%)$ & 44.4 & 83.1 & $66(71.7 \%)$ & - & - & - \\
\hline \multirow{2}{*}{ Adolescents ( $n=266$ ) } & McCarthy et al. ${ }^{10}$ & $34(48.5 \%)$ & $159(81.1 \%)$ & 47.8 & 81.5 & $193(72.5 \%)$ & 0.166 & 0.088 & 0.372 \\
\hline & Cole et al. ${ }^{12}$ & $24(34.2 \%)$ & $178(90.8 \%)$ & 57.1 & 79.4 & $202(75.9 \%)$ & - & - & - \\
\hline \multirow[t]{4}{*}{ Overall $(n=358)$} & Williams et al. ${ }^{8}$ & $45(48.3 \%)$ & $265(84.1 \%)$ & 51.7 & 82.2 & $268(74.8 \%)$ & 0.289 & 0.485 & 1.000 \\
\hline & Taylor et al. ${ }^{9}$ & $50(53.7 \%)$ & 210 (79.2\%) & 47.6 & 83 & $260(72.6 \%)$ & 0.109 & 0.091 & 0.497 \\
\hline & McCarthy et al. ${ }^{10}$ & 47 (50.5\%) & 211 (79.6\%) & $46.5 \%$ & $82.1 \%$ & $258(72.1 \%)$ & 0.200 & 0.106 & 0.397 \\
\hline & Cole et al. ${ }^{12}$ & $36(38.7 \%)$ & 232 (87.5\%) & 52.1 & 80.2 & $268(74.8 \%)$ & - & - & - \\
\hline
\end{tabular}

Abbreviations: BMI, body mass index; NPV, negative predictive value; PPV, positive predictive value; ROC, receiver operating characteristics; SENS, sensitivity; SPEC, specificity. ${ }^{\mathrm{a}} \chi^{2}$-test.

${ }^{6}$ Rate of subjects correctly classified.

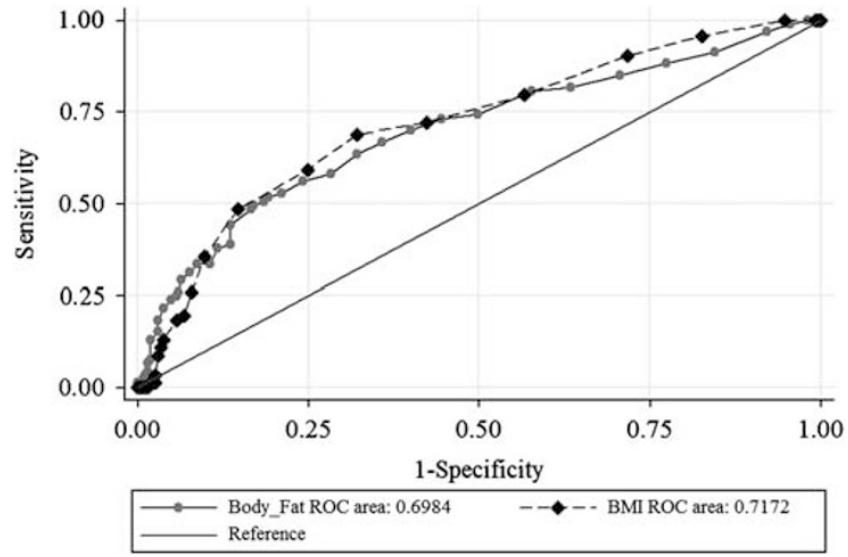

Figure 3 Graphical plots of the sensitivity and specificity in overall sample. ROC, receiver operating characteristic.

presence of EBP. However, this higher accuracy did not achieve statistical significance.

Area under curve has been used as a global measure of the performance of $\% \mathrm{BF}$ and BMI cutoffs. In our study, the area under curve values were low (data not shown), indicating the limited performance of both. The \%BF and BMI tables were highly specific, but insensitive for EBP screening, which agrees with Christofaro et al., ${ }^{22}$ who analyzed a large sample of adolescents of both genders and found decreased positive predictive value (male $=22 \%$ and female $=18 \%$ ). To adopt a more sensitive or specific \%BF cutoff is the researcher's choice, and depends on the desired function for these cutoffs. In population groups with a higher prevalence of the determined outcome, higher specificity seems more adequate, because it better excludes false-positive cases and prevents overtaxing the health system. On the other hand, an outcome of low prevalence requires a more sensitive tool, which increases the true-positive cases and, in turn, improves the probability of finding subjects in need of treatment.

In the case of EBP, an outcome of moderate/high prevalence with an increasing trend, higher specificity seems more appropriate. The three
\%BF cutoffs do not perform better than BMI in EBP screening, where true-positive, true-negative and efficiency of BMI were similar to the \%BF measured by DXA, independently of cutoffs.

In children and adolescents, early hypertension detection is important because it tracks into adulthood. Regarding hypertension screening, BMI was performed, as well as \%BF measured by DXA. BMI is determined by cheap anthropometric measurements, which can be carried out annually in schools and can be used as a simple screening tool, inserted into comprehensive public health strategies, focused on preventive actions. ${ }^{23}$ Another interesting finding is that, although obesity presents a great burden in the hypertension genesis, ${ }^{17}$ all analyzed cutoffs presented poor screening characteristics, indicating that without adequate cutoffs, $\% \mathrm{BF}$ monitoring may not allow for complete hypertension screening.

The strengths of our study include the use of DXA, a previously validated measure of $\% \mathrm{BF}$ in pediatric populations, ${ }^{24}$ with a relatively large sample size. However, its limitations must be clarified. The sample was composed only of boys, and it is unclear whether the results can be generalized to girls. The use of a single syndrome metabolic indicator (EBP values) and the measurement of BP values on a single day constitute the main limitations of the study. Additionally, when assessing adiposity in childhood and adolescence, even considering age adjustments, maturational stage should also be considered as a determinant or confounding variable and, therefore, the absence of this data constitutes a limitation of the study.

In summary, the results indicated that body fat estimated by DXA is strongly associated with EBP in pediatric populations, but the three analyzed body fat cutoffs have limited screening characteristics. Moreover, the BMI reference values proposed by Cole et al. ${ }^{12}$ are a more attractive tool for this function, as they show similar performance in EBP screening and are more suitable for large epidemiological studies because of their lower cost.

\section{CONFLICT OF INTEREST}

The authors declare no conflict of interest.

\section{ACKNOWLEDGEMENTS}

RAF received a postgraduate scholarship from CAPES (Coordenadoria de Aperfeiçoamento do Pessoal de Nível Superior). 
1 Wang Y, Monteiro C, Popkin BM. Trends of obesity and underweight in older children and adolescents in the United States, Brazil, China, and Russia. Am J Clin Nutr 2002; 75: 971-977.

2 Wang G, Dietz WH. Economic burden of obesity in youths aged 6-17 years: 1979-1999. Pediatrics 2002; 109: 1195-1200.

3 Chiolero A, Madeleine G, Gabriel A, Burnier M, Paccaud F, Bovet P. Prevalence of elevated blood pressure and association with overweight in children of a rapidly developing country. J Hum Hypertens 2007; 21: 121-127.

4 Salvadori M, Sontrop JM, Garg AX, Truong J, Suri RS, Mahmud FH, Macnab JJ, Clark WF. Elevated blood pressure in relation to overweight and obesity among children in a rural Canadian community. Pediatrics 2008; 122: 821-827.

5 McGavock JM, Torrance B, McGuire KA, Wozny P, Lewanczuk RZ. The relationship between weight gain and blood pressure in children and adolescents. Am J Hyperten 2007; 20: 1038-1044.

6 Fernandes RA, Freitas Júnior IF, Codogno JS, Christofaro DG, Monteiro HL, Roberto Lopes DM. Resting heart rate is associated with blood pressure in male children and adolescents. J Pediatr 2011; 158: 634-637.

7 Jafar TH, Islam M, Poulter N, Hatcher J, Schmid CH, Levey AS, Chaturvedi N. Children in South Asia have higher body mass-adjusted blood pressure levels than white children in the United States: a comparative study. Circulation 2005; 111: 1291-1297.

8 Williams DP, Going SB, Lohman TG, Harsha DW, Srinivasan SR, Webber LS, Berenson GS. Body fatness and risk for elevated blood pressure, total cholesterol, and serum lipoprotein ratios in children and adolescents. Am J Public Health 1992, 82: 358-363.

9 Taylor RW, Jones IE, Williams SM, Goulding A. Body fat percentages measured by dualenergy X-ray absorptiometry corresponding to recently recommended body mass index cutoffs for overweight and obesity in children and adolescents aged 3-18 y. Am J Clin Nutr 2002; 76: 1416-1421.

10 McCarthy HD, Cole TJ, Fry T, Jebb SA, Prentice AM. Body fat reference curves for children. Int J Obes (Lond) 2006; 30: 598-602.

11 Norton K, Olds T. Anthropometrica: A Textbook of body measurement for sports and health courses. UNSW Press: New South Wales, 1996

12 Cole TJ, Bellizzi MC, Flegal KM, Dietz WH. Establishing a standard definition for child overweight and obesity worldwide: international survey. BMJ 2000; 320: 1-6.

13 Christofaro DG, Casonatto J, Polito MD, Cardoso JR, Fernandes R, Gerage AM, Guariglia DA, Oliveira AR. Evaluation of the Omron MX3 Plus monitor for blood pressure measurement in adolescents. Eur J Pediatr 2009; 168: 1349-1354.
14 Pickering TG, Hall JE, Appel LJ, Falkner BE, Graves JW, Hill MN, Jones DH, Kurtz T, Sheps SG, Roccella EJ. Recommendations for blood pressure measurement in humans and experimental animals: Part 1: blood pressure measurement in humans: a statement for professionals from the Subcommittee of Professional and Public Education of the American Heart Association Council on High Blood Pressure Research. Hypertension 2005; 45: 142-161.

15 National High Blood Pressure Education Program Working Group on High Blood Pressure in Children and Adolescents. The fourth report on the diagnosis, evaluation, and treatment of high blood pressure in children and adolescents. Pediatrics 2004; 114: 555-576.

16 Moura AA, Silva MA, Ferraz MR, Rivera IR. Prevalence of high blood pressure in children and adolescents from the city of Maceió, Brazil. J Pediatr (Rio J) 2004; 80: 35-40.

17 Kotsis V, Stabouli S, Papakatsika S, Rizos Z, Parati G. Mechanisms of obesity-induced hypertension. Hypertens Res 2010; 33: 386-393.

18 Fernandes RA, Christofaro DG, Buonani C, Araujo LR, Kassab YK, Cardoso JR, Freitas Junior IF, Machado DR. The accuracy of national body fat cutoff levels in the prediction of elevated blood pressure among Brazilian male adolescents. J Trop Pediatr 2010; 56: 208-209.

19 Fernandes RA, Rosa CS, Silva CB, Bueno DR, Oliveira AR, Freitas Junior IF. Accuracy of different body mass index cutoffs to predict excessive body fat and abdominal obesity in adolescents. Rev Assoc Med Bras 2007; 53: 515-519.

20 Vitolo MR, Campagnolo PD, Barros ME, Gama CM, Ancona Lopez F. Evaluation of two classifications for overweight among Brazilian adolescents. Rev Saude Publica 2007; 41: 653-656.

21 Neovius M, Rasmussen F. Evaluation of BMI-based classification of adolescent overweight and obesity: choice of percentage body fat cutoffs exerts a large influence. The COMPASS study. Eur J Clin Nutr 2008; 62: 1201-1207.

22 Christofaro DG, Fernandes RA, Polito MD, Romanzini M, Ronque ER, Gobbo LA, Oliveira AR. A comparison between overweight cutoff points for detection of high blood pressure in adolescents. J Pediatr (Rio J) 2009; 85: 353-358.

23 Okuda M, Sugiyama S, Kunitsugu I, Hinoda Y, Okuda Y, Shirabe K, Yoshitake N, Hobara T. Use of body mass index and percentage overweight cutoffs to screen Japanese children and adolescents for obesity-related risk factors. J Epidemiol 2010; 20: 46-53.

24 Wells JC, Fuller NJ, Dewit O, Fewtrell MS, Elia M, Cole TJ. 4-component model of body composition in children: density and hydration of fat-free mass and comparison with simpler models. Am J Clin Nutr 1999; 69: 904-912. 